\title{
The Discrimination of Inrush Current from Internal Fault of Power Transformer based on EMD
}

\author{
Fanyuan Zeng, Qianjin Liu, Chao Shi \\ School of Electric Power, South China University of Technology,Guangzhou, China \\ Email: zeng_fanyuan@163.com
}

Received March, 2013

\begin{abstract}
The method is based on that the waveform of the inrush distorts seriously, while the fault current nearly keeps sinusoid. The complicated signal can be decomposed into a finite intrinsic mode functions (IMF) by the EMD, then define and compute the projection area on $\mathrm{X}$-axis of each $\mathrm{IMF}-S_{c i}$, the specific gravity of SIMF- $K_{c i}$, and the maximum of $K_{c i}-K_{\max }$. We can get a new scheme of transformer-protection based on comparing the difference between inrush and fault current. Theoretical analysis show that the method can precisely discriminate inrush and fault current, fault clearance time is about 20ms. Moreover, it is convenient to achieve and hardly be affect by not-periodic component.
\end{abstract}

Keywords: Inrush Current; Transformer Protection; HHT; EMD; IMF

\section{Introduction}

At present, the domestic transformer primary protection in power system configuration mainly uses second harmonic restraint principle and longitudinal differential protection based on current discontinuous corner braking principle. The long-term operating experience shows that the differential protection can not accurately distinguish the difference between the transformer internal faults and external faults, so the main contradiction is still focused on the identification of magnetizing inrush and internal fault.

\section{Empirical Mode Decomposition}

Empirical Mode Decomposition (EMD) can effectively identify magnetizing inrush and internal fault. EMD is suitable for the analysis of non-linear, non-stationary signal sequence with a high signal-to-noise ratio. The center of this technology is empirical mode decomposition, which can decompose complex signals into a finite number of intrinsic mode functions (IMF).The decomposed IMF component contains the local features signal of the different time scales of the original signal. The empirical mode decomposition method can make the non- stationary data become smooth and get the Hilbert transform spectrogram and the frequency of physical significance. Compared with the short-time Fourier transform and wavelet decomposition methods, this method is intuitive, direct, posterior and adaptive.

Basic principles of empirical mode decomposition:
In order to obtain the intrinsic mode functions, the data signal must be decomposed with EMD. So it is necessary to introduce the definition of the basic concepts of EMD decomposition process: IMF. This is the basis of the master of EMD method.

The intrinsic mode function must satisfy the following two conditions:

1) In the entire time range, the number of local extreme points and zero-crossing points of the function must be equal to, or up to a difference of one;

2) At any time, the average of the local maximum of the envelope (upper envelope) and the local minimum of the envelope (the envelope line) must be zero.

The first condition obviously has the similar requirements with traditional narrowband stationary Gaussian signal. For the second condition, it is a new concept which is the classic global requirements modifies localized requirements, so that the instantaneous frequency is no longer subject to the asymmetric waveform with unnecessary fluctuations. In fact, this condition should be "the local mean of the data is zero". However, for nonstationary data calculating the local mean involves the concept of the local time scale, which is difficult to define. Therefore, in the second condition, the envelope flocal maxima and local minimum values of the average envelope are instead of zero, which make the wave form of a signal locally symmetric. Huang et al study shows that, under normal circumstances, to use this instead of the physical significance of the instantaneous frequency is in line with the system studied. Intrinsic mode func- 
tions show the inherent vibration mode of data. Because of the intrinsic mode functions defined by the zero-crossing point of each vibration cycle, there is only one vibration mode with no other complex riding waves.

\section{Decomposition Process of EMD Method}

The EMD decomposition method is based on the following as assumptions:

1) Data must include at least two extreme values, a maximum value and minimum value; 2) Local time domain characteristics of the data is uniquely deter mined by the time scalebetweentheextreme points; 3) If data has an inflection point instead of extreme point, the decomposition results can be obtained by differentiating the data once or more times and integrating the extremum. The essence of this approach gets the intrinsic fluctuations mode by the characteristic time scale of the data, and then break down the data. This decomposition process can be vividly called "selecting” process.

Decomposition process: Find out all the maxima of the original data sequence $x(t)$ and cubic saline interpolattion function fitting form of the original data on envelope; Similarly, find out all the minimum point, and all of the minimum point formed by cubic spline interpolation function fitting the data under the envelope, the upper envelope and lower envelope means recorded as $m_{1}$, The original data sequence $x(t)$ by subtracting the average envelope $m_{1}$ obtain a new data sequence $h_{1}$.

$$
h_{1}=x(t)-m_{1}
$$

\section{Hilbert Spectra and Marginal Spectrum}

Huang et al proposed a new method for analysis of a signal systematically on the basis of the IMF and EMD, which called Hilbert-Huang Transform (HHT). It includes EMD and Hilbert spectral analysis methods corresponding to EMD.

First arbitrary signal s (t) is broken down into a finite number of IMF with EMD.

$$
s(t)=\sum_{j=1}^{n} c_{j}(t)+r(t)
$$

Then each IMF component is analyzed by Hilbert transform, and finally get the homeopathic frequency signal

$$
s(t)=\operatorname{Re} \sum_{i=1}^{n} a_{i}(t) e^{j \phi_{1}(t)}=\operatorname{Re} \sum_{i=1}^{n} a_{i}(t) e^{j \int \omega_{1}(t) d t}
$$

Here omitted the residual function $r_{n}(t)$, Re represent the real part. We call the right side of the above formula for the Hilbert spectrum, referred to as the Hilbert spectrum.

$$
H(\omega, t)=\operatorname{Re} \sum a_{i}(t) e^{j \int \omega_{1}(t) d t}
$$

Omitting the residual function $r_{n}(t)$ since it is a constant, or a monotonic function. Although $r_{n}(t)$ can be seen as a part of a long period wave, taking into account the long period of uncertainty, and signal the information contained in the high-frequency component, so we should do the omission processing. In the expansion of each component, the amplitude and phase is time variable, and the same signal s (t) of the Fourier transform expansions is

$$
s(t)=\sum_{i=1}^{\infty} a_{i} e^{i \omega_{1} t}
$$

This clearly shows that: Generalizing the Fourier expansion can not only increase the frequency of the signal, but also represents a variable frequency. New ways break the shackles of the Fourier transform.

$$
H(\omega)=\int_{-\infty}^{\infty} H(\omega, t) d t
$$

\section{Transformer Protection Based on EMD}

\subsection{Fundamental}

Under condition of no-load closing of a transformer, due to core saturation nonlinear magnetic properties, it may produce the magnetizing inrush comparable short-circuit current. In Figure 4-1(a), the line OABP is basic magnetizing curve of the transformer .The line OABP is replaced by a two-stage approximate magnetization of polyline OC and CP, and when closing power supply voltage is set : $u=U_{m} \sin (\omega t+a)$, without considering the transformer leakage reactance.

While transformer is no-load closing $(t=0)$, we can get the magnetizing inrush approximate expression as (4-1) show.

$$
i=\left\{\begin{array}{l}
\frac{U_{m}}{\omega L_{o c}}\left[\cos a-\cos (\omega t+a)+\frac{\phi_{r}}{\phi_{m}}\right] \\
\phi<\phi_{c} \\
\frac{U_{m}}{\omega L_{c p}}\left[\cos a-\cos (\omega t+a)+\frac{\phi_{r}-\phi_{c}}{\phi_{m}}\right]+i_{c} \\
\phi \geq \phi_{c}
\end{array}\right.
$$

In Equation (4-1), $\phi$ is core flux of the transformer; $\phi_{r}$ is remanence of transformer; $\phi_{m}$ is amplitude of the steady-state flux, rated conditions for rated flux amplitude.

For fault current waveform keep sinusoidal characteristics. In this paper, the basic idea of the identification principle is: Read a section of the differential waveform data f, IMF can be attained by EMD for $\mathrm{f}$, where we can look for all the leading IMF. If the number of leading IMF is 1,it is fault current; if the number is more than one, it is magnetizing inrush. 


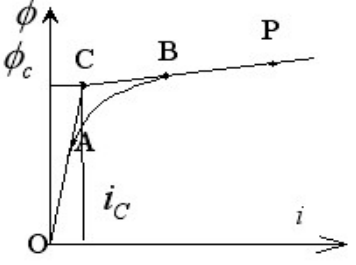

(a)

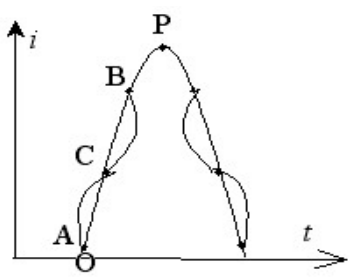

(b)
Figure 4.1. Top wave bump characteristics of the transformer.

\subsection{Specific Methods and Protection Criteria}

\subsubsection{Search IMF}

The dominant IMF is broken down into component of the IMF with a large amplitude .In order to search for the dominant IMF easily, this article defines IMF component $c_{i}$ on the horizontal axis of the projected area $S_{c i}$ as follows:

$$
S_{c i}=\int_{t_{o}}^{t_{n}}\left|c_{i}(t)\right| d t
$$

discretizing formula (4-2):

$$
S_{c i}=\sum_{k=1}^{n}\left|c_{i}(k) \Delta t\right|
$$

According to formula (4-3), the way to get the dominant IMF is: Calculate the various components $c_{i}$ of IMF, then $S_{\max }=\max \left\{S_{c i}, i=1 \ldots . n\right\}$, if the $S_{c i}$ of $c_{i}$ have a difference with $S_{\max }$ in $20 \%, c_{i}$ of IMF is the dominant IMF.

\subsubsection{Protection Criteria}

According to this identification principle, we define the proportion $c_{i}$ of component coefficient $K_{c i}$ is:

$$
K_{c i}=\frac{S_{c i}}{\sum_{k=1}^{n} S_{c k}+S_{r}}
$$

Assume the largest proportion of coefficients of IMF is $K_{\max }$,

$$
K_{\max }=\max _{i=1}^{n}\left\{K_{c i}\right\}=\frac{\max _{i=1}^{n}\left\{S_{c i}\right\}}{\sum_{k=1}^{n} S_{c k}+S_{r}}
$$

As it can be seen in formula (4-5), the value of $K_{\max }$ changes between $0 \sim 1$. When the differential current is fault current, since it contains only one dominant IMF, the value of $K_{\max }$ is very large, almost above 0.9 . When the differential current is inrush current, due to the presence of two or more similar to the proportion coefficient leading the IMF, the value of $K_{\max }$ is 0.5. Additionally, When the magnitude of the differential current data window is not greater than the maximum unbalanced cur- rent, the value of $K_{\max }$ is 0 .

Therefore, we can obtain protection criterion as (4-6) shows:

$$
K_{\max } \geq K_{z d}
$$

Define $K_{z d}=0.8$, respectively calculate three-phase differential current $K_{\max }$.

\subsection{Experimental Schematic and Waveform}

\subsubsection{Transformer Inrush Current Experiment}

Because of the limited laboratory equipment it is impossible to truly imitate inside the short-circuit experiments of transformer. Because the short circuit current is too large, and laboratory equipment can not stand the maximum short-circuit current, otherwise the short-circuit experiments will burn lines. Therefore, the present study apply inductive load to simulate the short circuit fault inside transformer.

\subsubsection{Experiment Resulting Waveform and Processing}

Experimental transformer no-load inrush current and load interterm short-circuit both cases, the two sets of waveforms, and a group of normal waveform. Since this article transformer experiment is the presence of harmonic components in the laboratory, during the test, so the experimental results from the waveform is not sinusoidal waveform, but presents the trend of a square wave.

Transformer inrush current experimental waveform graph is showed in Figure 4.2. Normal waveform is showed in Figure 4.3. Inrush current waveform is showed in Figure 4.4. Short circuit inside waveform is showed in Figure 4.5.

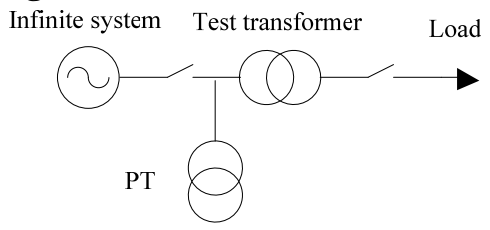

Figure 4.2. Transformer inrush current experimental waveform graph.

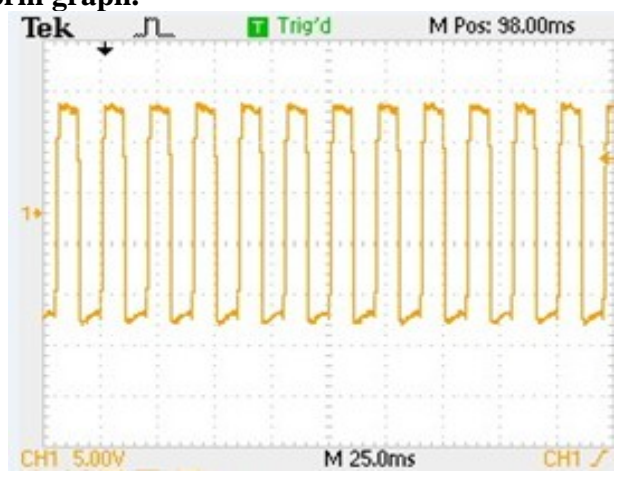

Figure 4.3. Normal waveform. 


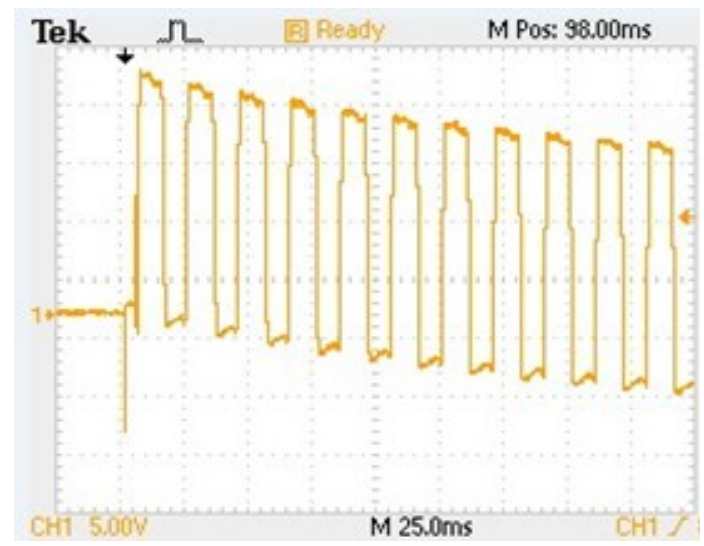

Figure 4.4. Inrush current waveform.

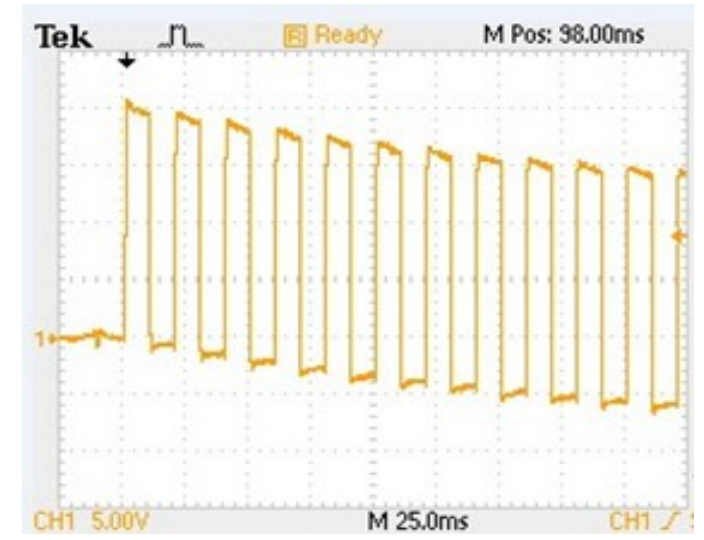

Figure 4.5. Short circuit inside waveform.

\section{Conclusions}

The processing method, fundamentally speaking, is based on the analysis of the three-phase current fundamental and higher harmonics, and then determines the algorithm of the higher harmonic content, but makes the method different from the conventional signal due to the role of the EMD methods of analysis. It is more convenient from the principles and experimental methods to distinguish normal airdrop and fault conditions, but there are also some shortcomings. The main reason is that the EMD algorithm is still not perfect in the border problem and envelope fitting: Firstly, there is not a suitable envelope exploded function resulting in fluctuations of $K_{\max }$; secondly, there is no particularly good boundary processing method.

\section{REFERENCES}

[1] T. Y. Li, Y. Zhao and N. Li, “A New Method for Power Quality Detection Based on HHT," Proceedings of the Csee, Vol. 25, No. 17, 2005, pp. 52-56

[2] Z. Ke, J. Jie and T. Q. Zhang, "Distinguishing Magnetizing Inrush Based on Characteristic of Equivalent Instantaneous Inductance,” Power System Protection and Control, 2010, pp. 12-16

[3] P. Liu, O. P. Malik, D. S. Chen, G. S. Hope and Y. Guo, "Improved Operation of Differential Protection of Power Transformers for Intemal Faults," IEEE Transactions on Power Delivery, Vo1. 7,1992.

[4] S. H. Jiao, W. S. Liu, J. F. Liu, Z. H. Zhang and Q. X. Yang, "A New Principle of Discrimination between Inrrush Current and Fault Current of Transformer Based on Wavelet," Proceedings of the CSEE, Jul. 1999, pp. $1-5$.

[5] J. A. Sykes and L. F. Morrison, “A Proposed Method of Harmonic Restraint Differential Protection of Transformers by Digital Computer," IEEE Transactions on Power Apparatus and Systems, Vol. PAS-91, pp. 1266-1273. doi:10.1109/TPAS.1972.293485

[6] F. M. Cao and P. P. Su, "The Application of Wavelet transform in Transformer's Differential Protection," Electric Power, Vol. 31, No. 11, 1998, pp. 21-24. 